\title{
Integrating academic analytics for supporting accreditation and international cooperation: TEC and UABCS experience
}

\author{
Mario Chacón-Rivas* \\ Agustín Francesa-Alfaro ${ }^{\dagger}$ \\ *machacon@itcr.ac.cr - Escuela de Computación \\ †afrancesa@itcr.ac.cr - TEC Digital \\ Instituto Tecnológico de Costa Rica \\ Cartago, Costa Rica
}

\author{
Mónica Carreño-León $\ddagger$ \\ Andrés Sandoval-Bringas ${ }^{\S}$ \\ Email: ${ }^{\ddagger}$ mcarreno@uabcs.mx \\ $\S_{\text {sandoval@uabcs.mx }}$ \\ Universidad Autónoma de Baja California Sur \\ Baja California Sur, México
}

\begin{abstract}
Information is the most important asset of organizations, currently, the fast, effective and accurate access is vital for strategic decision making and the organizations competitiveness. The education area is not the exception, so this article aims to share the experiences of collaboration between two Universities about learning analitycs and academic analytics tools, to motivate other universities to generate collaboration links. In this article, we refer the differences between learning analytics and academic analytics, the description of some tools developed at the Instituto Tecnológico de Costa Rica and the Universidad Autónoma de Baja California Sur, focused on these topics and the experiences of collaboration between both universities in two ways: sharing the current tools and how to develop new tools, capable of integrating with the infrastructure of each university in particular.
\end{abstract}

\section{Introducción}

Las necesidades de información de valor, concisa y resumida son día a día más necesarias en todos los contextos de organizaciones y de industria, por ello es que surge la teoría de analítica de la información o de datos. Esta teoría persigue facilitar información objetiva y precisa para soportar la toma de decisiones estratégicas y que permita mantener la competitividad de las organizaciones, mediante la aplicación de modelajes y métodos predictivos que van más allá de simples estadísticas descriptivas.

En el contexto de las universidades no es ajena la urgencia de contar con información precisa y efectiva para dar soporte a la toma de decisiones estratégicas, con miras a mejorar los procesos. Los procesos de gestión académica generalmente toman más del $80 \%$ del tiempo productivo de los académicos responsables de unidades o programas de formación universitarios. Además estos procesos de gestión académica se conocen, a partir de reuniones y conversaciones informales con criterio experto, que son repetitivos y atentan fuertemente contra la producción académica y la investigación.
También es evidente que las universidades se enfrentan a un mayor escrutinio por parte de los gobiernos, agencias de acreditación, estudiantes y padres; quienes de una u otra manera buscan calidad en los procesos y resultados. Es muy normal en la actualidad ver la tendencia de rendición de cuentas de las universidades en sus sitios web, publicando datos sobre sus logros, tiempos de graduación promedio, entre muchos otros ${ }^{1} 2$. Estos datos también pueden usarse para guiar la mejora interna de los procesos y quehacer académico. Como lo indican Campbell y Oblinger en [1] esta cultura de rendición de cuentas impone nuevas presiones a la educación superior, pero estas presiones también crean nuevas oportunidades para que las universidades reexaminen los procesos y herramientas que usan para la toma de decisiones.

Por otro lado, como se describe más adelante, existen soluciones de software existentes que se pueden compartir y adaptar a las universidades, pero, ¿Qué hacer cuando se quiere desarrollar un nuevo sistema, para diferentes universidades? Para atender esta situación, se plantea una propuesta de arquitectura de desarrollo que permita integrarse en diferentes plataformas.

\section{Contexto}

Las experiencias que se comparten en este trabajo son producto de la colaboración entre el Instituto Tecnológico de Costa Rica (TEC) y la Universidad Autónoma de Bajas California Sur (UABCS). Específicamente la colaboración se da entre el TEC Digital, unidad responsable de la plaforma de e-learning del TEC y el departamento Académico de Sistemas Computacionales de la UABCS. La colaboración entre ambas universidades se concreta en la integración de servicios desarrollados en las plataformas de gestión de los aprendizajes y en la plataforma de gestión de información de estudiantes.

1. https://www.tec.ac.cr/transparencia

2. http://www1.uabcs.mx/transparencia/ 
Además, se está proponiendo una arquitectura de desarrollo de software, con el fin de poder integrar nuevas soluciones a los LMS de cada universidad, de manera que se integren sin la necesidad de que la plataforma educativa sea la misma.

\subsection{Contexto TEC}

El TEC es una universidad estatal que cuenta con 46 años de fundada y actualmente imparte 28 carreras de pregrado, de las cuales 24 son ingenierías. Cuenta además con 3 programas de doctorado y 12 programas de maestría. El TEC se compone de una población estudiantil de 11.000 estudiantes y un cuerpo docente de alrededor de 800 personas, todos distribuidos geográficamente en 6 sedes. A nivel de información posee registros digitales de sus estudiantes, cuerpo docente y planes de estudio, historiales de progreso académico en bases de datos desde el año 1979. Emplea como plataforma de gestión del aprendizaje .LRN ${ }^{3}$, a la cual le ha ampliado los servicios por medio de más de 40 proyectos desarrollados durante una década. Estos proyectos han sido publicados en diversos medios y conferencias, se destacan algunos como [2], [3], [4], sin embargo para efectos de este trabajo nos concentraremos en los servicios que dan soporte a los procesos de gestión académica.

Los proyectos que dan soporte a la gestión académica son:

- Módulo de Directores: permite a los directores y coordinadores de programas o unidades académicas generar estadísticas sobre los índices de aprobación por curso. Integra también la visualización del progreso de los estudiantes en los planes de estudio. Además muestra estadísticas sobre el desempeño de los docentes en cuanto a porcentajes de aprobación y reprobación de sus estudiantes.

- Módulo de Proyección de Matrícula: realiza una proyección de cupos requeridos para un período siguiente (semestre, cuatrimestre, etc) basados en la información de la población actual de los cursos. Contempla porcentajes de aprobación históricos para los cursos, si los estudiantes tiene aprobados los requisitos y correquisitos de los cursos en proyección, como se puede apreciar en la figura 1.

- Módulo de Prematrícula: habilita una opción para que los estudiantes puedan indicar las intenciones de matrícula para el periodo siguiente. Esto permite un proceso de validación del mismo modo que el de proyección de matrícula.

- Módulo de Indicadores de Gestión de la Plataforma: presenta datos de uso de la plataforma, así como del uso de los servicios o aplicaciones agrupado por escuelas, carreras, cursos, e incluso comunidades (ver figura 2 utilización del servicio de evaluaciones). Esta información es de vital importancia para planificar crecimientos y demadas de espacios y equipos.

3. .LRN: plataforma basada en software libre, www.dotlrn.org

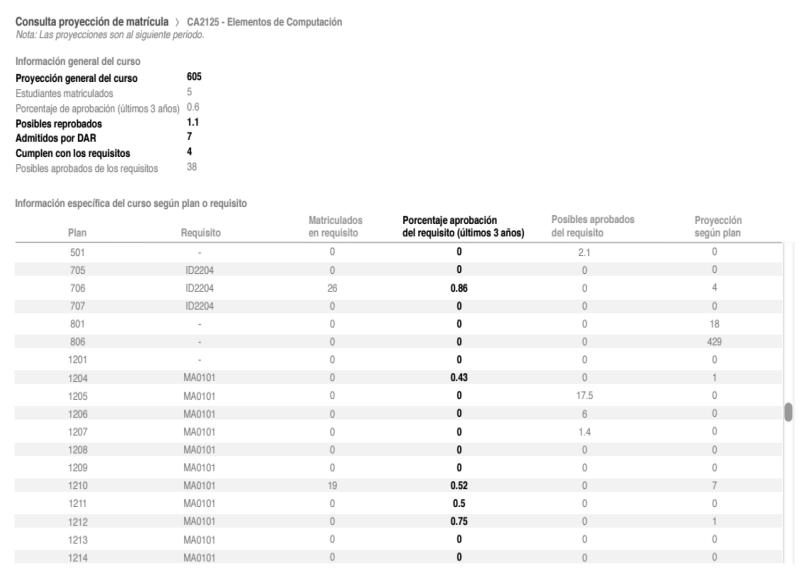

Figure 1. Módulo de proyección de Matrícula.

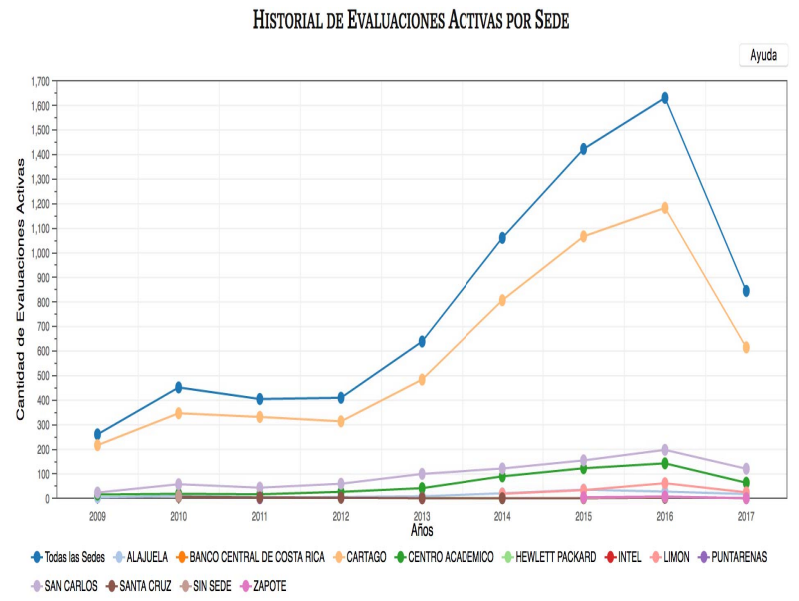

Figure 2. Módulo de indicadores de gestión de la plataforma.

- Módulo de Indicadores de Gestión Institucional: genera información estadística y de agrupamientos sobre los progresos de los estudiantes en los planes de estudios o carreras. Se emplea para generar información requerida por entes acreditadores y de gobierno. Este módulo junto con el anterior se basan en tecnologías de desarrollo de datawarehouse y creación de dashboards.

- Módulo de Trazabilidad: permite registrar las acciones o interacciones de los usuarios con la plataforma. Estas interacciones luego son empleadas para trabajar modelos de predicción de rendimiento académico ${ }^{4}$ basado en algoritmos como logistic regression, support vector machine, y neural networks. Además, de acuerdo con la interacción e información de otras herramientas, se pueden generar indicadores en tiempo real para el profesor, como se muestra en la figura 3.

4. Tesis de maestría en Ciencias de la Computación a presentarse en junio 2017. 


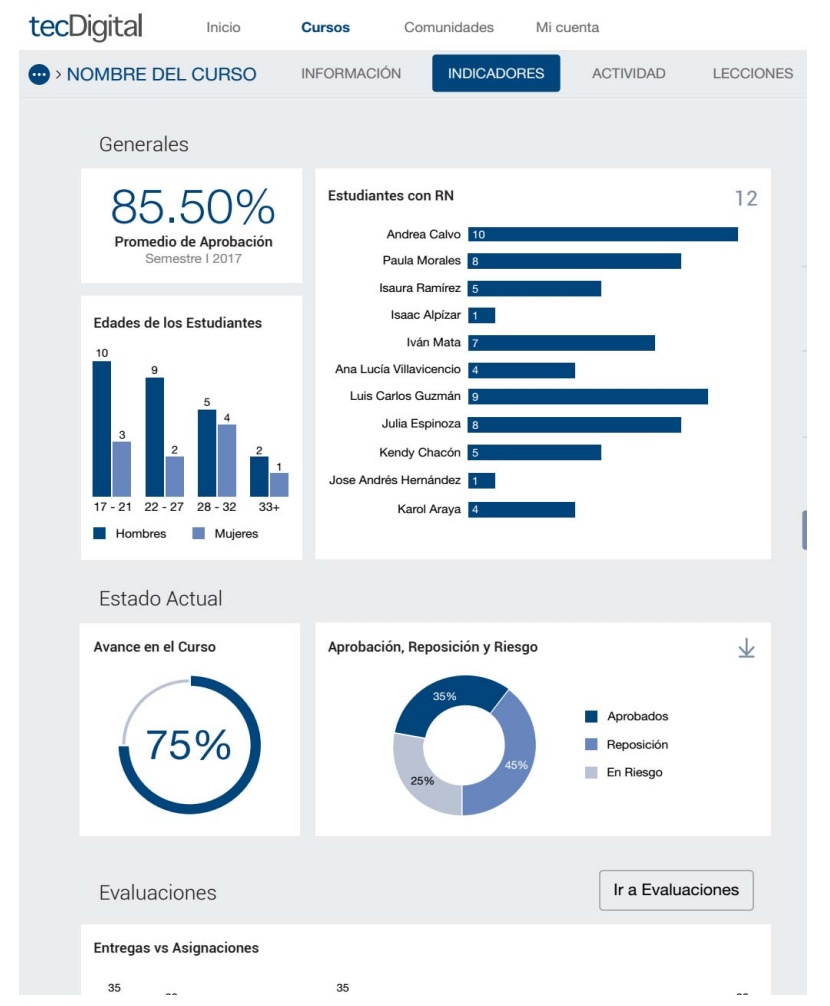

Figure 3. Módulo de Trazabilidad y estadísticas en tiempo real

\subsection{Contexto UABCS}

La UABCS es una universidad pública estatal con 41 años de antiguedad, la cual ofrece 28 carreras a nivel licenciatura, 9 maestrías y 3 doctorados.La UABCS cuenta con una población estudiantil de 7.000 estudiantes y una planta docente alrededor de 700 profesores-investigadores. La institución cuenta con el Sistema Integral de Información Administrativa (SIIA), el cual está compuesto por 3 módulos: Sistema de Control Escolar (SICOES), Sistema de Recursos Humanos (SIREHU) y Sistema de Administración y Finanzas (SIAFI). A través del Departamento Académico de Sistemas Computacionales (DASC) se han desarrollado diferentes proyectos informáticos con la finalidad de automatizar procesos académicos administrativos. Los módulos de tipo transaccional desarrollados como apoyo a la gestión académica-administrativa son:

- Módulo de Cargas y Horarios: permite la captura de la carga horaria para cada profesor, así como el horario asignado para la impartición de cada una de las asignaturas, esto se puede apreciar en la figura 4. A través de este módulo se asignan los espacios físicos para el desempeño de las actividades docentes.

- Módulo de Planeación de Asignaturas: permite a los profesores realizar la planeación semestral de las materias asignadas para un periodo determinado. A través de este módulo el profesor puede organizar en el tiempo los contenidos en función

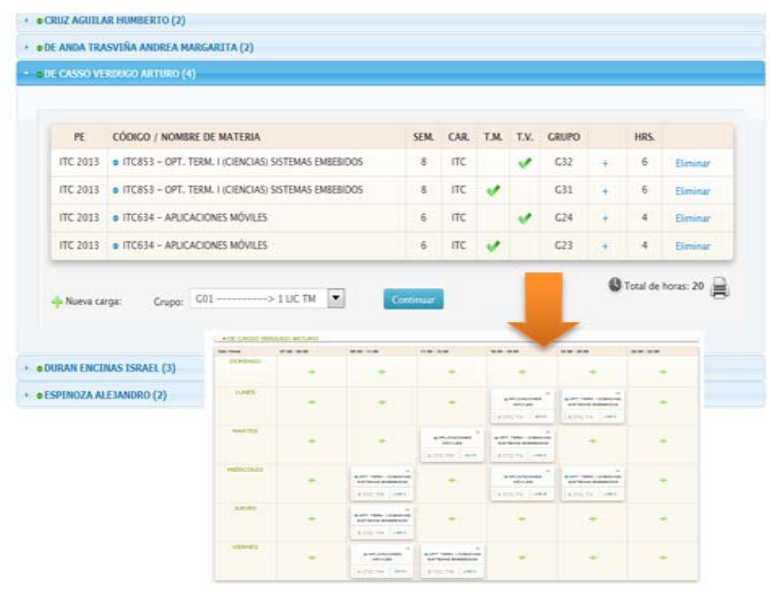

Figure 4. Interfaz de asignación de carga académica y horario a un profesor

a las habilidades que el estudiante deberá lograr de acuerdo a los aprendizajes esperados, así como los recursos necesarios, las dinámicas, prácticas, ejercicios y exámenes que permitan desarrollar el proceso de enseñanza- aprendizaje. En la figura $5 \mathrm{se}$ pueden apreciar las interfaces que permiten realizar la planeacion. Este módulo se encuentra ligado con el Módulo de Cargas y Horarios.

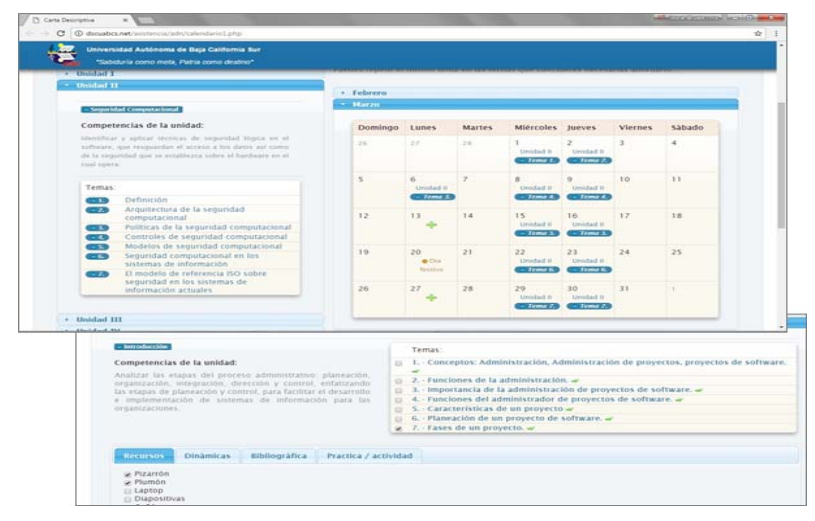

Figure 5. Interfaz de planeación de una asignatura

- Módulo de Registro de Asistencia y Avance Programático: permite a los profesores registrar los avances en la asignatura de acuerdo a lo planeado. A través de este módulo el profesor puede registrar el grado de avance en cada uno de los temas según lo propuesto en el Módulo de Planeación de Asignaturas. En la figura 6 se pueden apreciar las interfaces de este módulo.

- Módulo de Control de Asistencia de Alumnos y Evaluación: permite a los profesores registrar la asistencia a cada una de las sesiones programadas, así como el registro de las actividades y evaluaciones de cada uno de los alumnos. La información de este módulo es alimentada por el SICOES.

Por otro lado, se ha desarrollado un sistema para la 


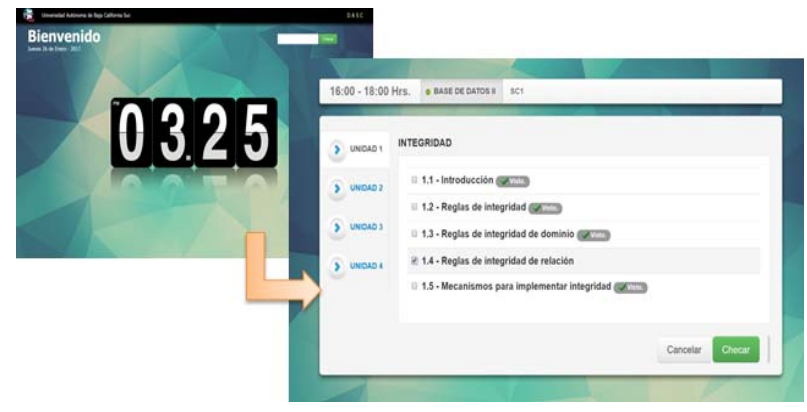

Figure 6. Interfaz para el registro de avance de una asignatura

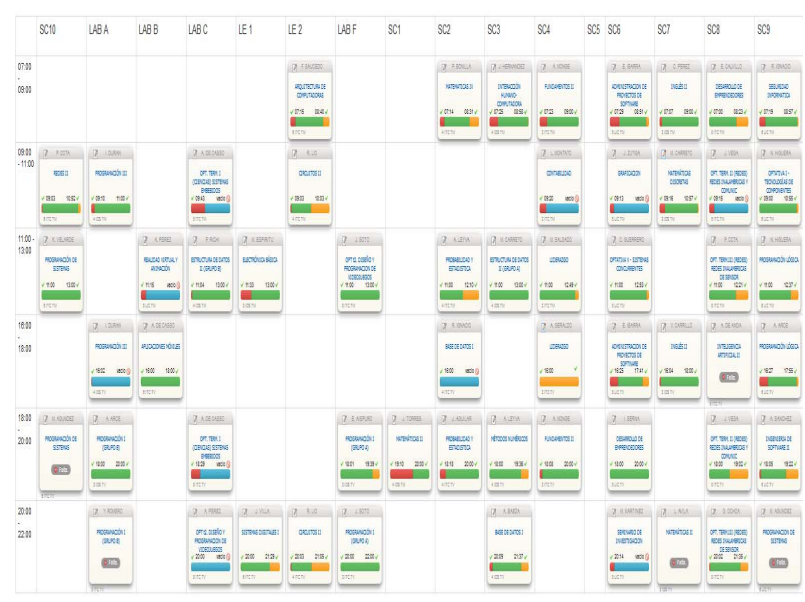

Figure 7. Detalle del registro de asistencia de profesores

toma de decisiones (DSS, por sus siglas en inglés) tomando como base el concepto de datawarehouse, que sirve de apoyo para el proceso de seguimiento académico que lleva a cabo cada uno de los departamentos académicos semestre tras semestre, para llevar a cabo la planeación académicaadmnistrativa. A manera de ejemplo, en la figura 8 se muestra el comportamiento de toda una cohorte generacional, a través de la agrupación de retículas individuales. Para esta generación en particular, se detectan cinco materias con atrasos. La materia con más rezago es la de Programación con solamente el 35\% de alumnos que han acreditado, Programación de Sistemas con el 40\%, Estructura de Datos II, Base de Datos I e Ingeniería del Software con el $45 \%$.

\section{Trabajos relacionados}

El proceso de toma de decisiones en las organizaciones se basa en (1) la intuición y (2) en emplear estrategias apoyadas en hipótesis que se respaldan y se buscan responder con datos y reportes históricos de la organización. Sin embargo, conforme las organizaciones van creciendo en volumen de información, los responsables de las decisiones requieren identificar patrones de comportamiento que se encuentran en las grandes cantidades de información. Para este último caso se emplean las técnicas de analítica de información.

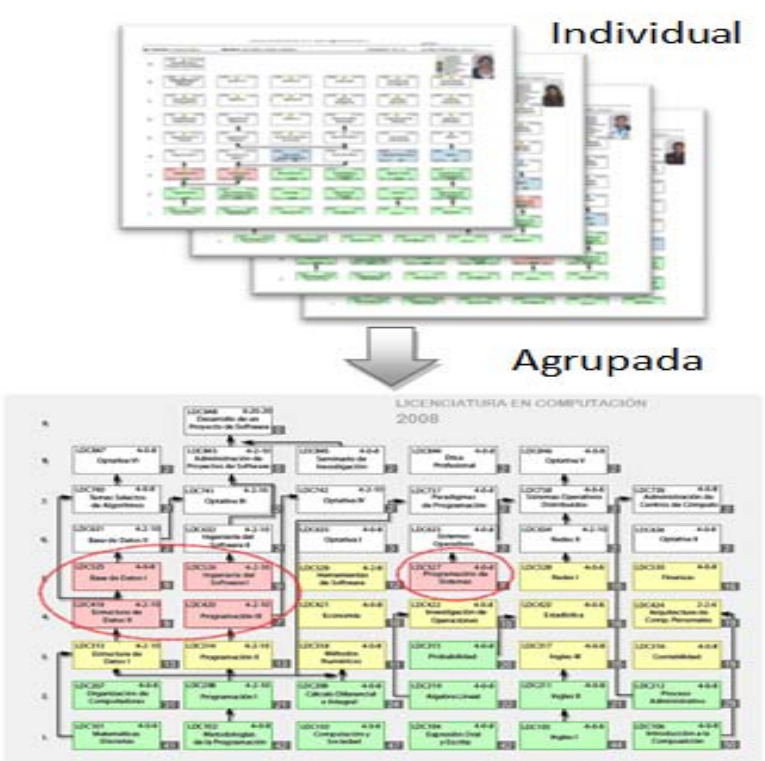

Figure 8. Ejemplo de preparación de información de una cohorte generacional

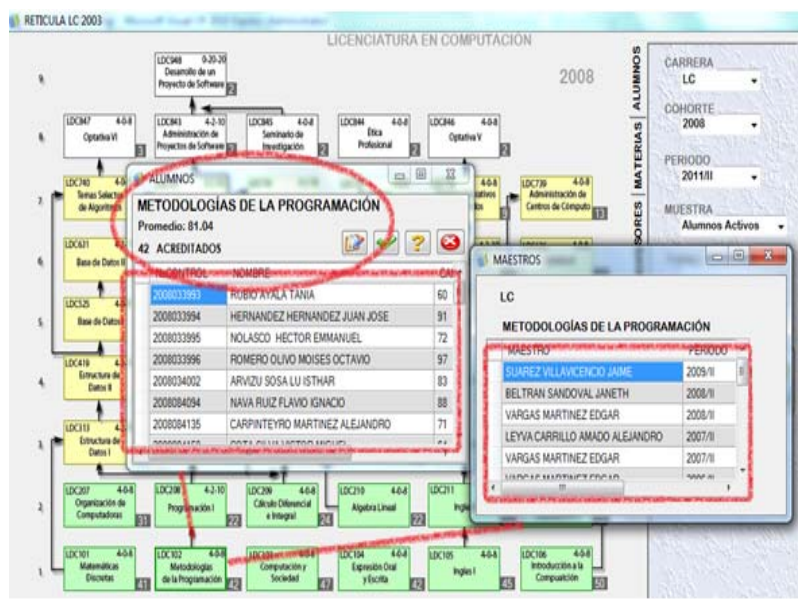

Figure 9. Detalle de cada una de las asignaturas

En el contexto de las universidades, la analítica académica permite relacionar técnicas estadísticas y modelos predictivos basados en volúmenes de datos con el fin de mejorar la toma de decisiones. Esto permitiría incluso predecir dificultades académicas de los estudiantes, con el fin de poder intervenir en forma temprana, potencialmente mejorar el proceso de enseñanza aprendizaje y finalmente impactar en la transparencia y rendición de cuentas [1], [5].

Es importante diferenciar el uso del término analítica del aprendizaje del término analítica académica. La analítica del aprendizaje es la aplicación de técnicas de analítica basadas en indicadores de comportamiento e interacción del estudiante en las plataformas, con el fin de lograr intervenciones docentes en la mejora de los cursos, personalización de ambientes, mejoras de los aprendizajes y evaluaciones así 
como su personalización. Una explicación clara de esto se puede ubicar en [6], [7].

En el contexto de este trabajo, emplearemos la analítica académica como la aplicación de las técnicas en los procesos de apoyo y mejora de la gestión académica [7], basando la toma de decisiones en información de mayor calidad. Se pretende la aplicación de estas técnicas para lograr un impacto significativo en la información empleada en los procesos de acreditación nacional e internacional de ambas universidades.

Diversos trabajos se han publicado relacionados con la aplicación de analítica académica. En [8] se presenta el proyecto Signals de la Universidad de Purdue, cuyo objetivo fue mejorar el éxito estudiantil dentro de un curso y así mejorar las tasas de retención y graduación de estudiantes en el tiempo planificado.

En [9] referencian un estudio en el que aplicando técnicas de agrupamiento, se descubre que en la Universidad de Michigan el 95\% de los accesos de los usuarios a la plataforma se centran en gestión de documentos y en acceso a mensajes o comunicación.

En [10] se presenta un estudio de la Universidad de Brigham Young el cual indica que 90\% de los accesos al LMS se realizan sobre solo 6 aplicaciones.

Finalmente, en [11] y en [12] se comentan varios proyectos, entre ellos el de la Escuela de Medicina de la Universidad de Wollongong. Otro proyecto es el modelo Trinkle 361 de la Universidad de Maryland, Baltimore, el cual alinea los objetivos estratégicos y la tecnología con la cultura organizacional.

Estos son ejemplos claros de estudios que conducen al uso de herramientas de analítica académica con el fin de reconsiderar la inversión en recurso humano, financieros y tecnológicos.

\section{Integración hacia la cooperación interna- cional}

Como parte de posibles formas de colaborar, se identificaron dos muy importantes, la colaboración a nivel de software existente para procesos de acreditación y otra para el desarrollo de nuevos sistemas, que sea ajusten a una arquitectura multiplataforma.

\subsection{Procesos de acreditación}

La integración propuesta está orientada a crear una plataforma de servicios de información eficiente y oportuna, basada en técnicas de procesamiento de la información que tienen ambas universidades.

El proceso de integración se basa en las siguientes actividades:

1) Identificación de las necesidades de información en cada universidad, este proceso conlleva al menos las siguientes actividades:

- Identificar el formato de información requerida.
- Identificar si existe la información en fuentes de datos propias a la universidad. En caso de no existir dicha información, verificar si es generada al menos en forma manual y si se mantienen históricos.

- Identificar los usuarios y organizaciones consumidores de dicha información.

- Identificar los responsables de la seguridad y mantenimiento de la información.

2) Identificación de los apoyos a nivel de autoridades en el compromiso y uso de la información y las tareas que esto conlleva para modificar y adaptar a las dimensiones estratégicas.

3) Identificación de las tecnologías disponibles para dar soporte a la integración y procesamiento de la información.

4) Identificación de disponibilidad de recurso humano con las competencias necesarias para abordar las etapas de integración de la información y luego del proceso mediante las técnicas de analítica de datos.

5) Rediseño de las fuentes de información y los formatos de presentación, con miras a que suplan las necesidades de ambas universidades.

6) Implementación de la integración y pruebas con los interesados.

De los procesos anteriores, los realmente medulares son los que se centran en el manejo de la información. Por esta razón es que el identificar las necesidades de información responde a priorizar los niveles estratégicos y de toma de decisiones. Un ejemplo de esto lo muestran los procesos de acreditación de programas de estudio o bien a nivel de la universidad. Según la experiencia de ambas universidades, las necesidades de información durante las acreditaciones son:

- Tiempos promedios de graduación.

- Cantidad de profesores a tiempo completo o equivalente.

- Cantidad de estudiantes.

- Distribución de estudiantes por profesor (o viceversa).

- Cantidad de estudiantes rezagados según plan de estudios.

- Porcentaje de uso de los servicios de la plataforma por curso y por profesor.

- Porcentajes de repitencia en cursos.

\subsection{Procesos de desarrollo de software}

En este propuesta, nos ubicamos al contexto de plataformas LMS y de software libre, con el fin de contribuir, independientemente de la plataforma que se use, si existe o no, con funcionalidades enfocadas a los procesos de enseñanza aprendizaje, capaces de ser integradas a los sistemas de cualquier institución Universitaria, ya que en la mayoría de casos, las necesidades de una institución a otra son muy similares, pero que un nuevo sistema se integre en todas no es sencillo. 
Esto se evidencia cuando se asiste a conferencias, en donde se muestra cómo una problemática relacionado a los procesos de enseñanza-aprendizaje son resueltos y con buenos resultados, pero que, por ciertos factores, no se puede integrar a otras plataformas; a pesar se tiene la intención de compartir las experiencias y hasta la solución de software.

Entre los factores que podrían afectar una integración en distintas plataformas, o para un proyecto multiplataforma se analizaron los siguientes:

- Motoros de base de datos y/o versiones.

- LMS, por ejemplo: Moodle y .LRN.

- Lenguajes de programación.

- Arquitecturas.

- Entre otros aspectos más técnicos.

Son por estas razones que se trabaja en modelar una arquitectura de software que permita conectarse por medio de un API a diferentes plataformas LMS de software libre, así como también en aplicaciones móviles.

La siguiente figura muestra la arquitectura propuesta.

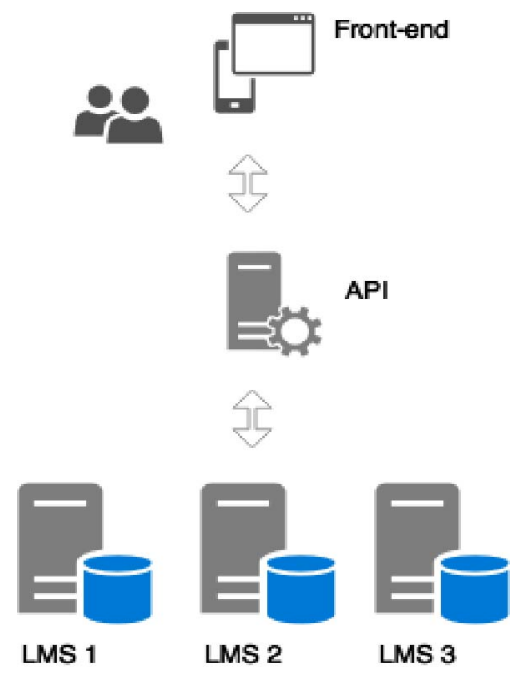

Figure 10. Aquitectura multiplataforma.

En la figrua anterior, en orden de abajo hacia arriba, se representan los diferentes LMS con sus respectivas bases de datos, los cuales podrían contener la misma información, pero almacenada de distinta manera.

La capa del medio representa el API, el cual es el mediador entre las bases de datos y el front-end, y es aquí donde se especifican las particularidades de cada plataforma a nivel de base de datos: dónde obtener la información, dónde almacenar, dónde actualizar y hasta eliminar.

Esta parte es la más importante y donde se necesitan los expertos en cada plataforma, de manera tal que de una fuente diversa de información, se procese y estandarice para ser mostrada en la interfaz gráfica y viceversa, desde la interfaz gráfica se envíe la información y se almacene en el lugar que corresponde en particular.
Por último, se muestra la capa del front-end, es en donde se da la interacción de los usuarios con el sistema, de manera que sin importar la plataforma, sería la misma manera de utilizar.

Esta arquitectura tiene múltiples beneficios al ser llevado a cabo de manera colaborativa, ente los analizados se encuentran:

- Mayor colaboración y alcance universal. Al desarrollar proyectos que se puedan utilizar en cualquier plataforma, se potencia la colaboración y el alcance del producto.

- Mayor retroalimentación.

- Comunidad internacional e-learning (multiplataforma).

- Reutilización de materiales, por ejemplo manuales, guías de uso, instrucciones, materiales de capacitación, entre otros.

- Investigación colaborativa, al tener las mismos servicios, se puede hacer investigación entre modelos de enseña-aprendizaje, por medio del análisis de los comportamientos en distintas universidades, o analizando un mayor número de datos.

- Potenciar la investigación y desarrollo del uso de las tecnologías en el proceso de enseñanza aprendizaje.

- Aumentar y estandarizar la accesibilidad en los sistemas.

- Entre otros.

Esta propuesta aún no ha sido probada, sin embargo, por experiencias previas conectando diferentes bases de datos y con el uso de software de Back-end as a Service se tienen conocimientos que indican que la solución es totalmente factible.

\section{Conclusiones y Trabajos Futuros}

La calidad de información y el uso que se le dé a la misma refleja de muchas maneras los niveles de madurez de las universidades, así como el tipo de gestión que emplean. Estos niveles son reflejados claramente en los procesos y logros de acreditación que poseen las mismas universidades. En el caso del TEC y la UABCS se hace evidente la generación y utilización de información precisa y orientada a la toma de decisiones.

La unificación de procesos y herramientas en torno a la calidad y procesos de acreditacino es tarea sencilla, sin embargo genera grandes beneficios a lo largo del tiempo. Cuando los procesos y herramientas a compartir y unificar provienen de diferentes universidades, conlleva una serie de actividades de aprendizaje y de homologación hasta de términos que enriquecen la cultura de cada organización. Esto no fue una excepción en el caso del trabajo conjunto del TEC y la UABCS.

Por otra parte, el empleo de instrumentos y aplicaciones que facilitan información para la toma de decisiones es frecuentemente visto por algunos grupos académicos o de apoyo como herramientas de control que atentan contra la flexibilidad y en algunos casos contra la libertad de 
cátedra. Esto genera resistencia al uso de los instrumentos que tienden a apoyar los procesos de toma de decisiones mejor fundamentados.

La experiencia generada en este proceso de integración ha revelado un reto organizacional y de ingeniería de software. Esto ha enriquecido las culturas desde la base conceptual, hasta enriquecer procesos de gestión y tecnológicos.

Además, es importante resaltar que en la era de la información, la colaboración, compartir experiencias y herramientas, es necesario; ya que en un ambiente competitivo, el acceso a la información precisa y disponible es esencial para la toma de decisiones. Una experiencia como esta acelera los procesos y da beneficios a las instituciones a corto plazo, incluyendo la validación de las propias herramientas y retroalimentación, la cual beneficia a todos.

Como trabajos futuros se pueden enumerar muchos, sin embargo los más relevantes en estas fases iniciales son los que se concentran en integración tecnológicas. Puntualmente desde compartir y conocer modelos de datos de las aplicaciones, arquitecturas involucradas, hasta ver detalles de documentación y comentarios de código fuentes, para el diseño y construcción del API.

Además, la propuesta de la arquitectura multiplataforma, se espera probar por medio del desarrollo de una aplicación móvil que permita realizar actividades de aprendizaje, ya sean prácticas, quices, exámenes, entre otras.

En esta experiencia se espera desarrollar un trabajo colaborativo multidisciplinar, en donde existirá la participación de asesores en tecnologías para la educación, ingenieros en computación, ingenieras en diseño con especialidad en comunicación visual, así como la participación de usuarios finales: profesores y estudiantes, de las diferentes universidades.

\section{Agradecimientos}

Los autores agradecen a las autoridades de ambas universidades que posibilitan la colaboración y que muestran la disposición a los trabajos conjuntos de crecimiento mutuo. Así como también a los equipo de trabajo y su dedicación para lograr los objetivos planteados.

\section{References}

[1] P. Campbell and D. Oblinger, "Academic analytics," EDUCAUSE review, vol. 42, no. 4, pp. 40-57, 2007.

[2] C. Garita and M. Chacon-Rivas, "Tec digital: A case study of an e-learning environment for higher education in costa rica," in 2012 International Conference on Information Technology Based Higher Education and Training (ITHET), 2012.

[3] M. Chacon-Rivas and C. Garita, "A successful oss adaptation and integration in an e-learning platform: Tec digital," in Open Source Software: Mobile Open Source Technologies. Springer, 2014, pp. 143-146.

[4] M. Chacon-Rivas and C.Garita, "Mobile course: Development of a mobile app to access university courses information," in Computing Conference (CLEI), 2013 XXXIX Latin American. IEEE, 2013, pp. $1-6$.
[5] A. Van Barneveld, K. Arnold, and J. Campbell, "Analytics in higher education: Establishing a common language," EDUCAUSE learning initiativeg, vol. 1, no. 1, pp. 1-11, 2012.

[6] M. Brown, "Learning analytics: moving from concept to practice," EDUCAUSE Learning Iniciative, 2012.

[7] P. Long and G. Siemens, "Fog penetrating: Analytics in learning and education," EDUCAUSE Review.

[8] K. Arnold, "Signals: applying academic analytics."

[9] P. Baepler and C. Murdoch, "Academic analytics and data mining in higher education."

[10] M. Griffiths and C. Graham, "Patterns of user activity in the different features of the blackboard cms across all courses for an academic year at brigham young university," MERLOT Journal of Online Learning and Teaching, p. 9, June 2009.

[11] K. D. Mattingly, M. C. Rice, and Z. L. Berge, "Learning analytics as a tool for closing the assessment loop in higher education," Knowledge Management \& E-Learning: An International Journal (KM\&EL), vol. 4, no. 3, pp. 236-247, 2012.

[12] U. bin Mat, N. Buniyamin, P. M. Arsad, and R. Kassim, "An overview of using academic analytics to predict and improve students' achievement: A proposed proactive intelligent intervention," in Engineering Education (ICEED), 2013 IEEE 5th Conference on. IEEE, 2013, pp. 126-130. 